\title{
The Centre for Internationalization and Usability: Enabling Culture-Centred Design for All
}

\author{
José Abdelnour-Nocera, Andy Smith, John Moore, Cecilia Oyugi, \\ Souleymane Camara, Malte Ressin, Sujan Shresta, and Alison Wiles \\ School of Computing and Technology, University of West London \\ St Mary's Road, Ealing. London, W5 5RF \\ \{Jose.Abdelnour-Nocera, Andy.Smith, John.Moore, Cecilia.Oyugi, \\ Souleymane.Camara, Malte.Ressin, Sujan. Shresta, \\ Alison.Wiles\} @uwl.ac.uk
}

\begin{abstract}
The Centre for Internationalisation and Usability within the School of Computing and Technology at The University of West London aims to enhance understanding of cultural differences in international software development. A particular focus is the development and usability of ICT products in a global market, both in terms of international software development and economic, community and social development. We host a number of researchers and $\mathrm{PhD}$ students working in topics such as usability evaluation and culture, sociotechnical participatory design, internationalization attitudes of software engineers, mobile learning and library cognitive design.
\end{abstract}

Keywords: internationalisation, usability, culture, sociotechnical, participatory design, empirical studies of software engineering.

\section{Research Challenges and Agenda}

The Centre for Internationalisation and Usability (CIU) aims to support software developers in building systems that meet all the needs of end users globally. A particular focus is concerned with software development in a global market and culture-centred design. Our research strategy is to enhance understanding and knowledge of cultural differences in international software development. There are two research challenges that need to be overcome to achieve this:

a) Computer science and interdisciplinary challenges that need to be addressed in order to provide tools, techniques and methods that can be used by software developers and interaction designers. We research the differing nature of these challenges in both the commercial and international development domains.

b) Institutional and governmental challenges to overcome resistance to change so that new tools, techniques and methods are actually implemented commercially. Implicit here is the requirement to collaborate with academics and practitioners internationally, both in promoting HCI and usability generally and localising the discipline itself, particularly in developing countries.

CIU has been successful in exploiting UK and EU funding streams in delivering its research strategy. We have accessed the EU Asia IT\&C programme to support 
usability research in both India and China. We have been the lead partner in the Sino European Systems Usability Network (SESUN) and the Indo European Systems Usability Partnership [1], helping to develop active and sustainable links between Asia and Europe. This has included collaborating on India's first international HCI conference and co-chairing a major conference in Beijing.

CIU was involved in the EPSRC funded VeSeL project - Village e-science for Life - addressing issues associated with the digital divide in Africa. VeSeL aims to fuse educational and environmental objectives to empower African village communities. Our work focused on socio-technical participatory design and the localization of usability methods [2]. More recently, CIU has begun to explore the use of emerging copyleft hardware technologies and how they might contribute to the fields of mobile learning and ICT for development (ICT4D) [3]. We believe that this open approach provides the opportunity for the community to directly influence the hardware roadmap of a device and thus generate sustainable solutions within markets of developing countries. In the e-learning domain, we are looking at university libraries' usability design from a socio-technical perspective. Our work with commercial usability agencies and clients such as Microsoft, HSBC, Siemens and Intercontinental has allowed us to develop a process model for developing usable cross-cultural websites [4]. Current commercial foci of our research concerns issues in relation to integration of internationalisation approaches within software development methods such as Agile [5] and in global software offshoring [6].

The impact of context and culture poses many challenges that cannot be exposed as a one-off evaluation in technology design. Decisions and actions in product design often result in the emergence of cultural and socio-technical implications and issues. CIU's research is aimed at the early identification of these in order to promote enhanced stakeholder participation and better product usability and user experience.

\section{References}

1. Smith, A., Joshi, A., Liu, Z., Bannon, L., Gulliksen, J., Li, C.: Institutionalising HCI in Asia. In: Baranauskas, C., Abascal, J., Barbosa, S.D.J. (eds.) INTERACT 2007. LNCS, vol. 4663, pp. 85-99. Springer, Heidelberg (2007)

2. Camara, S.B., Oyugi, C., Abdelnour-Nocera, J., Smith, A.: Augmenting usability: Cultural elicitation in HCI. In: Katre, D., Orngreen, R., Yammiyavar, P., Clemmensen, T. (eds.) HWID 2009. IFIP AICT, vol. 316, pp. 46-56. Springer, Heidelberg (2010)

3. Shrestha, S., Moore, J., Abdelnour-Nocera, J.: Low-cost hardware for ICT4D: what's right and what's left? Special Issue - IEEE Multidisciplinary Engineering Education Magazine (MEEM) 16(1) (2011)

4. Smith, A., Dunckley, L., French, T., Minocha, S., Chang, Y.: A process model for developing usable cross-cultural websites. Interacting with Computers 16, 63-91 (2004)

5. Ressin, M., Abdelnour-Nocera, J., Smith, A.: Of Code and Context: Collaboration Between Developers and Translators. In: Proceedings of CHASE 2011 at ICSE (2011)

6. Lee, D., Smith, A., Mortimer, M.: Cultural differences affecting quality and productivity in Western / Asian offshore software development. In: Proceedings of India HCI 2011 (2011) 